\title{
A New Theoretical Study of Quantum Atomic Energy Spectra for Lowest Excited States of Central (PIHOIQ) Potential in Noncommutative Spaces and Phases Symmetries at Plan's and Nanoscales
}

\author{
Abdelmadjid Maireche* \\ Laboratory of Physics and Material Chemistry, Physics department, Sciences Faculty, \\ University of M'sila-M'sila Algeria
}

(Received 23 March 2016; revised manuscript received 10 June 2016; published online 21 June 2016)

In this research paper, we consider full phase-space noncommutativity in the Schrödinger equation (SE), we apply Boopp's shift method and standard perturbation theory to the modified (SE) in order to obtain exactly new modified energy eigenvalues in noncommutative two dimensional real space-phase NC-2D: RSP for prolonged isotropic Harmonic oscillator plus inverse quadratic potential (PCIHOIQ potential) (central singular even-power potential (CSEP potential)) with novel two parts $2 \theta\left(\frac{b}{r^{4}}+\frac{c}{r^{6}}+\frac{d}{r^{8}}-\frac{a}{2}\right) L_{z}$ and $\frac{\bar{\theta} L_{z}}{2 m_{0}}$, it is observed that the new energy dependent with new atomic quantum numbers, we have also constructed the corresponding modified anisotropic Hamiltonian operator.

Keywords: Noncommutative space and phase, The singular even-power potential, Isotropic Harmonic oscillator plus inverse quadratic potential and Boopp's shift method.

DOI: $10.21272 /$ jnep.8(2).02027

PACS numbers: 11.10.Nx, 32.30. - r, 03.65. - w

\section{INTRODUCTION}

It is well-known that the central potentials in $D \geq 2$ dimensional spaces have been studied with various methods in different fields of nuclear physics, spectroscopy, quantum chemistry and many fields of sciences by solving the Schrödinger equation (SE), which plays a pivotal role in modern quantum physics and chemistry [1-25]. In particularly the central singular even-power potential (CSEP) that we study in the present work is useful to study the atomic physics and optical physics [26-28]. Recent years have witnessed the extensive exact solution for central potentials in noncommutative space-phase at Nano and Plank's scales [29-56]. The physical structure of new structure obtained by make the following translations:

$$
\begin{aligned}
& {\left[x_{i}, p_{j}\right]=i \delta_{i j} \rightarrow\left[\hat{x}_{i}^{*}, \hat{p}_{j}\right]=i \delta_{i j}} \\
& {\left[x_{i}, x_{j}\right]=0 \rightarrow\left[\hat{x}_{i}^{*}, \hat{x}_{j}\right]=i \theta_{i j}} \\
& {\left[p_{i}, p_{j}\right]=0 \rightarrow\left[\hat{p}_{i}^{*}, \hat{p}_{j}\right]=i \bar{\theta}_{i j}}
\end{aligned}
$$

Where the notation: $(*)$ denote to the new star product, the ordinary old product $f(x) g(x)$ between two arbitrary functions $f(x)$ and $g(x)$ will be $f(x) * g(x)[32-52]:$

$$
\begin{aligned}
& f(x) g(x) \rightarrow f(x) * g(x)=f(x) g(x) \\
& -\frac{i}{2} \theta^{\mu v} \partial_{\mu}^{x} f(x) \partial_{\nu}^{x} g(x)-\frac{i}{2} \bar{\theta}^{\mu v} \partial_{\mu}^{p} f(x) \partial_{\nu}^{p} g(x)
\end{aligned}
$$

The parameters $\theta^{\mu \nu}$ and $\bar{\theta}^{\mu \nu}$ are an antisymmetric real matrixes, the formalism of star product, Boopp's Shift method and the Seiberg-Witten map were played crucial roles in this new theory. The Boopp's shift method will be apply in present paper instead of solving the (NC-2D: RSP) with star product, the Schrödinger equation will be treated by using directly the following commutators [32-47]:

$$
\begin{aligned}
& {\left[\hat{x}_{i}^{*}, \hat{p}_{j}\right]=i \delta_{i j} \rightarrow\left[\hat{x}_{i}, \hat{p}_{j}\right]=i \delta_{i j}} \\
& {\left[\hat{x}_{i}^{*}, \hat{x}_{j}\right]=i \theta_{i j} \rightarrow\left[\hat{x}_{i}, \hat{x}_{j}\right]=i \theta_{i j}} \\
& {\left[\hat{p}_{i}^{*}, \hat{p}_{j}\right]=i \bar{\theta}_{i j} \rightarrow\left[\hat{p}_{i}, \hat{p}_{j}\right]=i \bar{\theta}_{i j}}
\end{aligned}
$$

Where the new operators $\hat{x}_{i}$ and $\hat{p}_{i}$ in (NC-2D: RSP) are depended with ordinary operator $x_{i}$ and $p_{i}$ from the following projections relations $(c=\hbar=1)$ [49]:

$$
\begin{array}{ll}
\hat{x}=x-\frac{\theta}{2} p_{y} & \text { and } \quad \hat{y}=y+\frac{\theta}{2} p_{x} \\
\hat{p}_{x}=p_{x}+\frac{\bar{\theta}}{2} y & \text { and } \quad \hat{p}_{y}=p_{y}-\frac{\bar{\theta}}{2} x
\end{array}
$$

For two dimensional spaces-phases, it is easy to verify the following important results:

$$
\begin{aligned}
& {\left[\hat{x}, \hat{p}_{x}\right]=\left[\hat{y}, \hat{p}_{y}\right]=i,} \\
& {[\hat{x}, \hat{y}]=i l_{N C}^{2} \quad \text { and } \quad\left[\hat{p}_{x}, \hat{p}_{y}\right]=\bar{l}_{N C}^{2}}
\end{aligned}
$$

\footnotetext{
*abmaireche@gmail.com
} 
Where $\left(l_{N C}^{2}, \bar{l}_{N C}^{2}\right) \equiv\left(\theta \equiv \theta_{12}, \bar{\theta} \equiv \bar{\theta}_{12}\right), l_{N C}^{2}$ is the square of space cell scale, this research paper based essentially on our previously works [32-49]. The objective to this paper is to extend our work, nonrelativistic Atomic spectrum for companied Harmonic oscillator potential and its inverse in both NC-2D: RSP [37] to include new two terms $\left(\frac{c}{r^{4}}+\frac{d}{r^{6}}\right)$ in ordinary two dimensional spaces on based to the paper of D. Shi-Hai [25]. This paper is structured as follows: in next section we briefly present the basic (CSEP) in ordinary two dimensional spaces. Section 3 is devoted to studying the 2-dimensional space-phase modified (SE) with modified (CPIHOIQ) potential; we derive the deformed Hamiltonian of Hydrogen atom including modified spin-orbital interaction and new Zeeman effect, we apply the ordinary perturbation theory to deduce the non relativistic modifications energy levels of electron corresponding new modified potential produced by the effect spin-orbital interaction and modified Zeeman effect. In section 4 we summarize the global energy spectra for (MSEP) corresponding lowest excitations states and we rebating the same results on based to another form to the perturbation potential. Finally, we give our concluding remarks in section 5 .

\section{THE (CSEP) POTENTIAL IN ORDINARY TWO DIMENSIONAL SPACES}

In polar coordinates $(r, \phi)$, the ordinary (SE) with singular even-power potential or prolonged isotropic Harmonic oscillator plus inverse quadratic potential (CPIHOIQ) potential $V_{c s e p}(r)=\left(a r^{2}+\frac{b}{r^{2}}\right)+\left(\frac{c}{r^{4}}+\frac{d}{r^{6}}\right)$, which consider the sum of isotropic Harmonic oscillator plus inverse quadratic potential $\left(V(r)=a r^{2}+\frac{b}{r^{2}}\right)$ and two new terms $\left(\frac{c}{r^{4}}+\frac{d}{r^{6}}\right)$, at low energy, is expressed as [25]:

$$
\begin{aligned}
& \left(-\frac{1}{2 m_{0}}\left(\frac{1}{r} \frac{\partial}{\partial r}\left(r \frac{\partial}{\partial r}\right)+\frac{1}{r^{2}} \frac{\partial^{2}}{\partial \phi^{2}}\right)+a r^{2}+\frac{b}{r^{2}}+\frac{c}{r^{4}}+\frac{d}{r^{6}}\right) \times \\
& \times \Psi(r, \phi)=E \Psi(r, \phi)
\end{aligned}
$$

Where $E,(a, b, c$ and $d)$ are the nonrelativistic energy of the system and real numbers, respectively. The method of separation of variable has been applied in reference [25]:

$$
\Psi(\vec{r})=\frac{R_{m}(r)}{\sqrt{r}} \Phi(\phi)
$$

The eq. (7) accepts a solution for the angular function $\Phi(\phi)$ and a radial function $R_{m}(r)$, respectiv1ely as follows [25]:

$$
\begin{aligned}
& \Phi(\phi)=\exp ( \pm i m \phi) \\
& \text { and } \\
& R_{m}(r)=\exp \left(p_{m}(r)\right) \sum_{n=0} a_{n} r^{2 n+\delta} \text { where } m=0,1,2 \ldots
\end{aligned}
$$

Where the function $p_{m}(r)$ is given by [25]:

$$
p_{m}(r)=\frac{1}{2} \alpha r^{2}+\frac{1}{2} \beta^{\prime} r^{4}
$$

Here $\alpha, \beta$ and $\delta$ are determined by [25]:

$$
\beta^{\prime 2}=d, \quad \alpha^{2}=a, \quad \delta=\frac{3}{2}+\mu \quad \text { and } \mu=\frac{c}{2 \sqrt{d}}
$$

The normalized eignenfunctions $\left(\Psi^{(0)}(\vec{r}), \Psi^{(1)}(\vec{r})\right)$ and the energy eigenvalue of the (CSEP) potential corresponding ground state and first excited states ( $\left.E_{0}, E_{1}\right)$, respectively, are as given by [25]:

$$
\left\{\begin{array}{l}
\Psi^{(0)}(\vec{r})=a_{0} r^{\delta-\frac{1}{2}} \exp \left(-\frac{\sqrt{a} r^{2}+\sqrt{d} r^{-2}}{2}\right) \\
E_{0}=\sqrt{a}(4+2 \mu),
\end{array}\right.
$$

And

$$
\left\{\begin{array}{l}
\Psi^{(1)}(\vec{r})=\left(a_{0}+a_{1} r^{2}\right) r^{\delta-\frac{1}{2}} \exp \left(-\frac{\sqrt{a} r^{2}+\sqrt{d} r^{-2}}{2}\right) \exp ( \pm i \phi) \\
E_{1}=\sqrt{a}(8+2 \mu)
\end{array}\right.
$$

\section{THE MODIFIED (CPIHOIQ) POTENTIAL IN (NC-2D: RSP )}

In this section, we shell give the deformed Hamiltonian operator $H_{n c-s e p}$ for modified (CPIHOIQ) potential in (NC-2D: RSP), it satisfied by the following translations [32-49]:

$$
H_{\text {csep }}=\frac{\vec{P}^{2}}{2 m_{0}}+\hat{V}_{\text {sep }}(r) \rightarrow H_{N C-\text { sep }}=\frac{\hat{\vec{p}}^{2}}{2 m_{0}}+\hat{V}_{\text {sep }}(\hat{r})
$$

Where $\hat{\vec{p}}^{2}$ and $\hat{V}_{\text {csep }}(\hat{r})$ are the modified of the kinetic energy and new operator of the (PIHOIQ) potential in (NC-2D: RSP), which allow us to obtaining the deformed Schrödinger equation:

$$
\left(\frac{\hat{\vec{p}}^{2}}{2 m_{0}}+\hat{V}_{\text {csep }}(\hat{r})\right) * \hat{\Psi}(\hat{r})=E_{N C} \hat{\Psi}(\hat{r})
$$

Here $E_{N C}$ is the noncommutative energy; the Boopp's shift method is usually used to solving the deformed Schrödinger equation with the central potential as follows:

$$
\begin{aligned}
& \left(\frac{\hat{\vec{p}}^{2}}{2 m_{0}}+\hat{V}_{\text {csep }}(\hat{r})\right) * \hat{\Psi}(\hat{r})=E_{N C} \hat{\Psi}(\hat{r}) \rightarrow \\
& \left(\frac{\hat{\vec{p}}^{2}}{2 m_{0}}+V_{\text {csep }}(\hat{r})\right) \Psi(\hat{r})=E_{N C} \Psi(\hat{r})
\end{aligned}
$$

$V_{\text {csep }}(\hat{r})$ Is the new modified potential as a function of operator $\hat{r}$ :

$$
V_{\text {csep }}(\hat{r})=a \hat{r}^{2}+\frac{b}{\hat{r}^{2}}+\frac{c}{\hat{r}^{4}}+\frac{d}{\hat{r}^{6}}
$$


Applying the relation (4) to find the following terms in NC-2D: RSP:

$$
\hat{r}^{2}=a r^{2}-a \theta L
$$

After a straightforward calculation, we can prove that, the three important terms: $\frac{b}{\hat{r}^{2}}, \frac{c}{\hat{r}^{4}}$ and $\frac{d}{\hat{r}^{6}}$ can be takes the following expressions:

$$
\begin{aligned}
& \frac{b}{\hat{r}^{2}}=\frac{b}{r^{2}}+\frac{2 b \theta}{r^{4}} L_{z} \\
& \frac{c}{\hat{r}^{4}}=\frac{c}{r^{4}}+\frac{2 c \theta}{r^{6}} L_{z} \\
& \frac{d}{\hat{r}^{6}}=\frac{d}{r^{6}}+\frac{2 d \theta}{r^{6}} L_{z}
\end{aligned}
$$

For the kinetic terms, on based to our reference [49], we can write:

$$
\hat{\vec{p}}^{2}=\vec{p}^{2}+\bar{\theta} L_{z}
$$

Where $L_{z}=x p_{y}-y p_{x}$ and we have also $L_{x}=L_{y}=0$ regarding the explicit values of $\hat{r}^{2}$ and $\hat{\vec{p}}^{2}$ in NC-2D: RSP, we can deduce that the roles $\theta$ and $\bar{\theta}$ are oppositely and satisfied the symmetries of space-phase, furthermore the new operators $\hat{x}_{i}$ and $\hat{p}_{i}$ are playing similarly rolls in the transformations like the transformations of electric and magnetic fields in special relativity. Now, substituting the obtained results (16.2) into eq. (15), to get the deformed spatial potential $V_{\text {csep }}(\hat{r})$ as follows:

$$
V_{\text {csep }}(\hat{r})=a r^{2}+\frac{b}{r^{2}}+\frac{c}{r^{4}}+\frac{d}{r^{6}}+H_{\text {sep } 1-p e r}(r)
$$

Where $H_{c s e p 1-p e r}(r)$ is given by:

$$
H_{c s e p 1-p e r}(r)=\theta\left(-a+\frac{2 b}{r^{4}}+\frac{2 c}{r^{6}}+\frac{2 d}{r^{8}}\right) L_{z}
$$

The global new part of the modified potential $H_{\text {csep-per }}(r)$ is the sum $H_{\text {sep } 1-p e r}(r)$ with the second part of eq. (16.3):

$$
H_{\text {csep-per }}(r)=2 \theta\left(\frac{b}{r^{4}}+\frac{c}{r^{6}}+\frac{d}{r^{8}}-\frac{a}{2}\right) L_{z}+\frac{\bar{\theta}}{2 m_{0}} L_{z}
$$

Then, the new NC-2D Hamiltonian will be written as follows for modified (CPIHOIQ) potential:

$$
H_{N C}=H_{c s e p}+H_{c s e p-p e r}(r)
$$

Here $H_{\text {sep }}$ is the usual commutative Hamiltonian in ordinary two dimensional spaces:

$$
\begin{aligned}
& H_{\text {csep }}=-\frac{1}{2 m_{0}}\left(\frac{1}{r} \frac{\partial}{\partial r}\left(r \frac{\partial}{\partial r}\right)+\frac{1}{r^{2}} \frac{\partial^{2}}{\partial \phi^{2}}\right)+ \\
& +a r^{2}+\frac{b}{r^{2}}+\frac{c}{r^{4}}+\frac{d}{r^{6}}
\end{aligned}
$$

Considering the noncommutativity as a small perturbation on the structure of the phase space, the real parameters ( $\theta$ and $\bar{\theta}$ ) are taken very small and our calculations are taken up to the first order in $\theta$ and $\bar{\theta}$. The new added part $H_{\text {sep-per }}(r)$ is proportional with the small non-commutative two parameters $\theta$ and $\bar{\theta}$, which we consider as a perturbative term. Furthermore, we can rewrite it to the equivalent physical form for modified (CPIHOIQ) potential:

$$
H_{\text {csep-per }}(r)=\left(2 \theta f(r)+\frac{\bar{\theta}}{m_{0}}\right) \vec{S} \vec{L}
$$

Where the radial scalar function $f(r)$ is given by:

$$
f(r)=\frac{2 b}{r^{4}}+\frac{2 c}{r^{6}}+\frac{2 d}{r^{8}}-a
$$

This allows us to write the perturbative term $H_{\text {csep-per }}(r)$ as:

$$
H_{c s e p-p e r}(r)=\left(\theta\left(\frac{2 b}{r^{4}}+\frac{2 c}{r^{6}}+\frac{2 d}{r^{8}}-a\right)+\frac{\bar{\theta}}{2 m_{0}}\right) \vec{G}^{2}
$$

Where $\vec{G}^{2} \equiv \vec{J}^{2}-\vec{L}^{2}-\vec{S}^{2}, J$ is the total momentum, $L$ is the angular momentum and $\mathrm{S}$ is the spin momentum. The operator $\left(2 \theta f(r)+\frac{\bar{\theta}}{m_{0}}\right) \vec{S} \vec{L}$ traduces physically the coupling between spin and orbital momentum. Then, the corresponding NC Hamiltonian $H_{n c-c s e p}$ will be:

$$
\begin{aligned}
& H_{n c-c s e p}=-\frac{1}{2 m_{0}}\left(\frac{1}{r} \frac{\partial}{\partial r}\left(r \frac{\partial}{\partial r}\right)+\frac{1}{r^{2}} \frac{\partial^{2}}{\partial \phi^{2}}\right)+V_{\text {csep }}(r)+ \\
& +\left(\theta f(r)+\frac{\bar{\theta}}{2 m_{0}}\right) \vec{G}^{2}
\end{aligned}
$$

After a straightforward calculation, we can show that the radial part $R_{m}(r)$ of the (MSE) for a solved quantum bound state problem in (NC-2D: RSP) is given by:

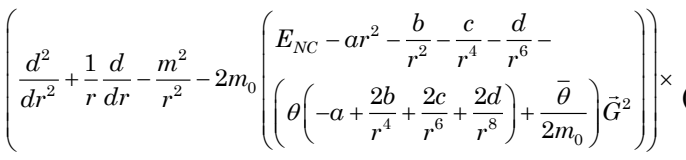

$$
\begin{aligned}
& \times R_{m}(r)=0
\end{aligned}
$$

It's well known that, the operators: $\left(L_{x}, L_{y}, L_{z}\right)$ are symmetry generators satisfying the Lie algebra and therefore, $\left(\vec{J}^{2}, \vec{L}^{2}, \vec{S}^{2}\right.$ and $\left.J_{z}\right)$ is complete set of commuting observables in ordinary quantum mechanics. 
Then the operator $\vec{G}^{2}$ will have two eigenvalues: $L\left(j=l+\frac{1}{2}, l, s\right) \quad$ and $\quad L\left(j=l-\frac{1}{2}, l, s\right) \quad$ corresponding $j=l+\frac{1}{2}$ (spin up) and $j=l-\frac{1}{2}$ (spin down), respectively [32-49]:

$$
\begin{aligned}
& L\left(j=l+\frac{1}{2}, l, s\right)=\left(l+\frac{1}{2}\right)\left(l+\frac{3}{2}\right)-l(l+1)-\frac{3}{4} \equiv L_{U} \\
& L\left(j=l-\frac{1}{2}, l, s\right)=\left(l-\frac{1}{2}\right)\left(l+\frac{1}{2}\right)-l(l+1)-\frac{3}{4} \equiv L_{D}
\end{aligned}
$$

Then, we can form a diagonal matrix $H_{n c-c s e p}(r)$ of or$\operatorname{der}(2 \times 2)$ with elements $H_{u p-c s e p}$ and $H_{\text {dow-csep }}$, respectively, as follows:

$$
\begin{aligned}
& H_{\text {up-csep }}=-\frac{1}{2 m_{0}}\left(\frac{1}{r} \frac{\partial}{\partial r}\left(r \frac{\partial}{\partial r}\right)+\frac{1}{r^{2}} \frac{\partial^{2}}{\partial \phi^{2}}\right)+ \\
& +a r^{2}+\frac{b}{r^{2}}+\frac{c}{r^{4}}+\frac{d}{r^{6}}+ \\
& +\left(\theta\left(\frac{2 b}{r^{4}}+\frac{2 c}{r^{6}}+\frac{2 d}{r^{8}}-a\right)+\frac{\bar{\theta}}{2 m_{0}}\right) L_{U} \\
& \text { if } j=l+\frac{1}{2} \text { :for- spin-up }
\end{aligned}
$$

And

$$
\begin{aligned}
& H_{\text {dow-csep }}=-\frac{1}{2 m_{0}}\left(\frac{1}{r} \frac{\partial}{\partial r}\left(r \frac{\partial}{\partial r}\right)+\frac{1}{r^{2}} \frac{\partial^{2}}{\partial \phi^{2}}\right)+ \\
& +a r^{2}+\frac{b}{r^{2}}+\frac{c}{r^{4}}+\frac{d}{r^{6}} \\
& +\left(\theta\left(\frac{2 b}{r^{4}}+\frac{2 c}{r^{6}}+\frac{2 d}{r^{8}}-a\right)+\frac{\bar{\theta}}{2 m_{0}}\right) L_{D} \\
& \text { if } j=l-\frac{1}{2} \text { :for- spin- down }
\end{aligned}
$$

\subsection{The Modifications to the Energies Levels Produced by Spin-orbital Effect for Modified (CPIHOIQ) Potential}

The main goal to this subsection is reach to the energies of ground and first excited states: $E_{N U}$ and $E_{N D}$ of an electron with spin up and spin down, respectively, for modified (CPIHOIQ) potential in NC-2D: RSP:

$$
E_{N U, N D}=\left(E_{0}, E_{1}\right)+E_{U, D}
$$

Where $\left(E_{0}, E_{1}\right)$ is the energy for ground and first excited states in ordinary 2-dimensional space while $E_{U}$ and $E_{D}$ are the modifications to the energy levels, associated with spin up and spin down at first order of ( $\theta$ and $\bar{\theta}$ ), to obtain those corrections, we need to apply the perturbation theory:

$$
E_{U, D}=L_{U, D} \int \Psi^{(p) *}(\vec{r})\left(\theta f(r)+\frac{\bar{\theta}}{2 m_{0}}\right) \Psi^{(p)}(\vec{r}) r d r d \phi(29)
$$

3.1.1 The Modifications to the Energies Levels Produced by Spin-orbital Effect for Ground State for Modified (CPIHOIQ) Potential

The non-commutative modifications of the energy levels for (CPIHOIQ) potential associated with spin up and spin down, in the first order of ( $\theta$ and $\bar{\theta})$ corresponding to the stationary state $\left(E_{0 U}\right.$ and $\left.E_{0 D}\right)$, are determined using Eqs. (11.1), (23) and (29) to obtain:

$$
\begin{aligned}
& E_{0 U, 0 D}=2 \pi \theta L_{U, D} \int_{0}^{+\infty}\left[a_{0} r^{\delta-\frac{1}{2}} \exp \left(-\frac{\sqrt{a} r^{2}+\sqrt{d} r^{-2}}{2}\right)\right]^{2} f(r) r d r \\
& +\frac{\bar{\theta} \pi}{m_{0}} L_{U, D} \int_{0}^{+\infty}\left[a_{0} r^{\delta-\frac{1}{2}} \exp \left(-\frac{\sqrt{a} r^{2}+\sqrt{d} r^{-2}}{2}\right)\right]^{2} r d r
\end{aligned}
$$

This can be simplified to:

$$
\begin{aligned}
& E_{0 U}=2 \pi L_{U} a_{0}^{2} \theta \sum_{\mu=1}^{4} S_{e p 0}{ }^{\mu}+\frac{\bar{\theta} \pi}{m_{0}} a_{0}^{2} L_{U} S_{e p 0}{ }^{5} \\
& E_{0 D}=2 \pi L_{D} a_{0}^{2} \theta \sum_{\mu=1}^{4} S_{e p 0}{ }^{\mu}+\frac{\bar{\theta} \pi}{m_{0}} a_{0}^{2} L_{D} S_{e p 0}{ }^{5}
\end{aligned}
$$

Where the 5 - factors $S_{e p 0}{ }^{\mu}(\mu=\overline{1.5})$ are given by:

$$
\begin{aligned}
& S_{e p 0}{ }^{1}=-a \int_{0}^{+\infty} r^{(2 \delta+1)-1} \exp \left(-\sqrt{a} r^{2}-\sqrt{d} r^{-2}\right) d r, \\
& S_{e p 0}{ }^{2}=2 b \int_{0}^{+\infty} r^{(2 \delta-3)-1} \exp \left(-\sqrt{a} r^{2}-\sqrt{d} r^{-2}\right) d r \\
& S_{e p 0}{ }^{3}=2 c \int_{0}^{+\infty} r^{(2 \delta-5)-1} \exp \left(-\sqrt{a} r^{2}-\sqrt{d} r^{-2}\right) d r, \\
& S_{e p 0}{ }^{4}=2 d \int_{0}^{+\infty} r^{(2 \delta-7)-1} \exp \left(-\sqrt{a} r^{2}-\sqrt{d} r^{-2}\right) d r
\end{aligned}
$$

And

$$
S_{e p 0}^{5}=\int_{0}^{+\infty} r^{(2 \delta+1)-1} \exp \left(-\sqrt{a} r^{2}-\sqrt{d} r^{-2}\right) d r
$$

Where $\beta \equiv \sqrt{a}$ and $\gamma \equiv \sqrt{d}$, now, it's useful to applying the following special integration $[57,58]$ :

$$
\int_{0}^{+\infty} x^{\nu-1 .} \exp \left(-\beta x^{p}-\gamma x^{-p}\right) d x=\frac{2}{p}\left(\frac{\gamma}{\beta}\right)^{\frac{v}{2 p}} K_{v / p}(2 \sqrt{\beta \gamma})
$$

Where $K_{v / p}$ denote to the Bateman's function, $\operatorname{Re} l(\beta)\rangle 0$ and $\operatorname{Re} l(\gamma>0)$. After straightforward calculations, we can obtain the explicitly the results:

$$
\begin{aligned}
& S_{e p 0}{ }^{1}=-a\left(\frac{d}{a}\right)^{\frac{2 \delta+1}{8}} K_{(2 \delta+1) / 2}\left(2(a d)^{1 / 4}\right), \\
& S_{e p 0}{ }^{2}=2 b\left(\frac{d}{a}\right)^{\frac{2 \delta-3}{8}} K_{(2 \delta-3) / 2}\left(2(a d)^{1 / 4}\right), \\
& S_{e p 0}{ }^{3}=2 c\left(\frac{d}{a}\right)^{\frac{2 \delta-5}{8}} K_{(2 \delta-5) / 2}\left(2(a d)^{1 / 4}\right), \\
& S_{e p 0}{ }^{4}=2 d\left(\frac{d}{a}\right)^{\frac{2 \delta-7}{8}} K_{(2 \delta-7) / 2}\left(2(a d)^{1 / 4}\right)
\end{aligned}
$$




$$
S_{e p 0}^{5}=\left(\frac{d}{a}\right)^{\frac{2 \delta+1}{8}} K_{(2 \delta+1) / 2}\left(2(a d)^{1 / 4}\right)
$$

Substituting eq. (35) and (36) into eq. (30) leads to write $E_{0 U}$ and $E_{0 D}$ as:

$$
\begin{aligned}
& E_{0 U}=L_{U}\left\{\theta K\left(a, d, a_{0}\right)+\bar{\theta} \bar{K}\left(a, d, a_{0}\right)\right\} \\
& E_{0 D}=L_{D}\left\{\theta K\left(a, d, a_{0}\right)+\bar{\theta} \bar{K}\left(a, d, a_{0}\right)\right\}
\end{aligned}
$$

Where the two factors $K\left(a, d, a_{0}\right)$ and $\bar{K}\left(a, d, a_{0}\right)$ are given by:

$$
K\left(a, d, a_{0}\right)=2 \pi a_{0}^{2} \sum_{\mu=1}^{4} S_{e p 0}{ }^{\mu}
$$

And

$$
\bar{K}\left(a, d, a_{0}\right)=\frac{\pi}{m_{0}} a_{0}^{2} L_{D} S_{e p 0}^{5}
$$

\subsubsection{The Modifications to the Energies Levels Produced by Spin-orbital Effect for First Excited States of Modified (CPIHOIQ) Potential}

In order to get the non-commutative first-order (in $\theta$ and $\bar{\theta}$ ) modification of the energy levels $E_{1 U}$ and $E_{1 D}$, for (MSEP) associated with spin up and spin down, respectively, corresponding to the first excited states, we use the Eqs. (11.2), (23) and (29) to obtain:

$$
\begin{aligned}
& \frac{E_{1 U}}{2 \pi L_{U}}=\int_{0}^{+\infty}\left[\left(a_{0}+a_{1} r^{2}\right) r^{\delta-\frac{1}{2}} \exp \left(-\frac{\sqrt{a} r^{2}+\sqrt{d} r^{-2}}{2}\right)\right]^{2}\left(\theta f(r)+\frac{\bar{\theta}}{2 m_{0}}\right) r d r \\
& \frac{E_{1 D}}{2 \pi L_{D}}=\int_{0}^{+\infty}\left[\left(a_{0}+a_{1} r^{2}\right) r^{\delta-\frac{1}{2}} \exp \left(-\frac{\sqrt{a} r^{2}+\sqrt{d} r^{-2}}{2}\right)\right]^{2}\left(\theta f(r)+\frac{\bar{\theta}}{2 m_{0}}\right) r d r
\end{aligned}
$$

The above relations can be simplified to the following forms:

$$
\begin{aligned}
& \frac{E_{1 U}}{2 \pi L_{U}}=\int_{0}^{+\infty}\left\{\begin{array}{l}
A_{1} \theta r^{2 \delta}+A_{2} \theta r^{2 \delta-4}+A_{3} \theta r^{2 \delta-6}+A_{4} \theta r^{2 \delta-8} \\
+A_{5} \theta r^{2 \delta+2}+A_{6} \theta r^{2 \delta-2}+A_{7} \theta r^{2 \delta+4} \\
+\frac{\bar{\theta}}{2 m_{0}}\left\{A_{8} r^{2 \delta}+A_{9} r^{2 \delta+4}+A_{10} r^{2 \delta+2}\right\}
\end{array}\right\} \times \\
& \times \exp \left(-\sqrt{a} r^{2}-\sqrt{d} r^{-2}\right) d r
\end{aligned}
$$

And

$$
\begin{aligned}
& \frac{E_{1 D}}{2 \pi L_{D}}=\int_{0}^{+\infty}\left\{\begin{array}{l}
A_{1} \theta r^{2 \delta}+A_{2} \theta r^{2 \delta-4}+A_{3} \theta r^{2 \delta-6}+A_{4} \theta r^{2 \delta-8} \\
+A_{5} \theta r^{2 \delta+2}+A_{6} \theta r^{2 \delta-2}+A_{7} \theta r^{2 \delta+4} \\
+\frac{\bar{\theta}}{2 m_{0}}\left\{A_{8} r^{2 \delta}+A_{9} r^{2 \delta+4}+A_{10} r^{2 \delta+2}\right\}
\end{array}\right\} \times \\
& \times \exp \left(-\sqrt{a} r^{2}-\sqrt{d} r^{-2}\right) d r
\end{aligned}
$$

Where $A_{i}(i=\overline{1,10})$, are determined from the relations:

$$
\begin{aligned}
& A_{1}=-a a_{0}^{2}+2 b a_{1}^{2}, A_{2}=2 b a_{0}^{2}+4 c a_{1} a_{0}+2 d a_{1}^{2} \\
& A_{3}=2 c a_{0}^{2}+4 d a_{1} a_{0}, A_{4}=2 d a_{0}^{2} \\
& A_{5}=-2 a a_{1} a_{0}, A_{6}=4 b a_{1} a_{0}+2 c a_{1}^{2} \\
& A_{7}=a a_{1}^{2}, A_{8}=a_{0}^{2}, A_{9}=2 a_{1} a_{0} \text { and } \mathrm{A}_{10}=a_{1}^{2}
\end{aligned}
$$

Substituting eq. (41) into eq. (40.1) and (40.2) leads to:

$$
\begin{aligned}
& E_{1 U}=L(j=l+s, l, s)\left\{2 \pi \theta A_{\mu} S_{e p 1}{ }^{\mu}+\pi \frac{\bar{\theta}}{m_{0}} A_{v} S_{e p 1}{ }^{\nu}\right\} \\
& E_{1 D}=L(j=l-s, l, s)\left\{2 \pi \theta A_{\mu} S_{e p 1}{ }^{\mu}+\pi \frac{\bar{\theta}}{m_{0}} A_{v} S_{e p 1}{ }^{\nu}\right\}
\end{aligned}
$$

We have used the Einstein notations (sum with indices $\mu=\overline{1,7}$ and $v=\overline{8,10})$, the factors $S_{e p 1}{ }^{\mu}(\mu=\overline{1,10})$, are given by:

$$
\begin{aligned}
& S_{e p 1}{ }^{1}=\int_{0}^{+\infty} r^{(2 \delta+1)-1} \exp \left(-\sqrt{a} r^{2}-\sqrt{d} r^{-2}\right) d r, \\
& S_{e p 1}{ }^{2}=\int_{0}^{+\infty} r^{(2 \delta-3)-1} \exp \left(-\sqrt{a} r^{2}-\sqrt{d} r^{-2}\right) d r, \\
& S_{e p 1}{ }^{3}=\int_{0}^{+\infty} r^{(2 \delta-5)-1} \exp \left(-\sqrt{a} r^{2}-\sqrt{d} r^{-2}\right) d r, \\
& S_{e p 1}{ }^{4}=\int_{0}^{+\infty} r^{(2 \delta-7)-1} \exp \left(-\sqrt{a} r^{2}-\sqrt{d} r^{-2}\right) d r, \\
& S_{e p 1}{ }^{5}=\int_{0}^{+\infty} r^{(2 \delta+3)-1} \exp \left(-\sqrt{a} r^{2}-\sqrt{d} r^{-2}\right) d r, \\
& S_{e p 1}{ }^{6}=\int_{0}^{+\infty} r^{(2 \delta-1)-1} \exp \left(-\sqrt{a} r^{2}-\sqrt{d} r^{-2}\right) d r, \\
& S_{e p 1}{ }^{7}=\int_{0}^{+\infty} r^{(2 \delta+5)-1} \exp \left(-\sqrt{a} r^{2}-\sqrt{d} r^{-2}\right) d r
\end{aligned}
$$

And

$$
\begin{aligned}
& S_{e p 1}^{8}=\int_{0}^{+\infty} r^{(2 \delta+1)-1} \exp \left(-\sqrt{a} r^{2}-\sqrt{d} r^{-2}\right) d r, \\
& S_{e p 1}^{9}=\int_{0}^{+\infty} r^{(2 \delta+5)-1} \exp \left(-\sqrt{a} r^{2}-\sqrt{d} r^{-2}\right) d r, \\
& S_{e p 1}^{10}=\int_{0}^{+\infty} r^{(2 \delta+3)-1} \exp \left(-\sqrt{a} r^{2}-\sqrt{d} r^{-2}\right) d r
\end{aligned}
$$

Applying, the special integral represented by eq. (34), we obtain the following results:

$$
\begin{gathered}
S_{e p 1}^{1}=\left(\frac{d}{a}\right)^{\frac{2 \delta+1}{8}} K_{v / p}\left(2(a d)^{1 / 4}\right), S_{e p 1}{ }^{2}=\left(\frac{d}{a}\right)^{\frac{2 \delta-3}{8}} K_{(2 \delta-3) / 2}\left(2(a d)^{1 / 4}\right), \\
S_{e p 1}^{3}=\left(\frac{d}{a}\right)^{\frac{2 \delta-5}{8}} K_{(2 \delta-5) / 2}\left(2(a d)^{1 / 4}\right), S_{e p 1}{ }^{4}=\left(\frac{d}{a}\right)^{\frac{2 \delta-7}{8}} K_{(2 \delta-7) / 2}\left(2(a d)^{1 / 4}\right), \\
S_{e p 1}{ }^{5}=\left(\frac{d}{a}\right)^{\frac{2 \delta+3}{8}} K_{(2 \delta+3) / 2}\left(2(a d)^{1 / 4}\right), S_{e p 1}{ }^{6}=\left(\frac{d}{a}\right)^{\frac{2 \delta-1}{8}} K_{(2 \delta-1) / 2}\left(2(a d)^{1 / 4}\right), \\
\text { and } S_{e p 1}{ }^{7}=\left(\frac{d}{a}\right)^{\frac{2 \delta+5}{8}} K_{(2 \delta+5) / 2}\left(2(a d)^{1 / 4}\right)
\end{gathered}
$$

And 


$$
\begin{aligned}
& S_{e p 1}^{8}=\left(\frac{d}{a}\right)^{\frac{2 \delta+1}{8}} K_{(2 \delta+1) / 2}\left(2(a d)^{1 / 4}\right), S_{e p 1}{ }^{9}=\left(\frac{d}{a}\right)^{\frac{2 \delta+5}{8}} \times \\
& \times K_{(2 \delta+5) / 2}\left(2(a d)^{1 / 4}\right), \\
& \text { and } S_{e p 1}^{10}=\left(\frac{d}{a}\right)^{\frac{2 \delta+3}{8}} K_{(2 \delta+3) / 2}\left(2(a d)^{1 / 4}\right)
\end{aligned}
$$

Now, we turn to the non-commutative modification of the energy levels $E_{1 U}$ and $E_{1 D}$ for (MSEP) will be determined from the relations:

$$
\begin{aligned}
& E_{1 U}(j, l, s, \theta, \bar{\theta})=L\left(j=l+\frac{1}{2}, l, s=1 / 2\right) \times \\
& \times\left\{\begin{array}{l}
2 \pi \theta A_{\mu} S_{e p 1}{ }^{\mu}(\mu=\overline{1,7}) \\
+\pi \frac{\bar{\theta}}{m_{0}} A_{v} S_{e p 1}{ }^{v}(v=\overline{8,10})
\end{array}\right\}
\end{aligned}
$$

And

$$
E_{1 D}(j, l, s, \theta, \bar{\theta})=L_{D}\left\{\begin{array}{l}
2 \pi \theta A_{\mu} S_{e p 1}{ }^{\mu}(\mu=\overline{1,7}) \\
+\pi \frac{\bar{\theta}}{m_{0}} A_{v} S_{e p 1}{ }^{v}(v=\overline{8,10})
\end{array}\right\}
$$

Let us summarize the obtained results of energies ( $\left.E_{N 0}, E_{N 1}\right)$ corresponding the effect of spin-orbital perturbation operator associated with spin up and spin down in the first order of two infinitesimal parameters $\theta$ and $\bar{\theta}$ for the stationary state and the first excited states, in NC-2D: RSP as follows:

$$
\begin{aligned}
& E_{N 0}=\sqrt{a}(4+2 \mu)+ \\
& + \begin{cases}L_{U}\left\{\theta K\left(a, d, a_{0}\right)+\bar{\theta} \bar{K}\left(a, d, a_{0}\right)\right\} & j=l+1 / 2(48.1) \\
L_{D}\left\{\theta K\left(a, d, a_{0}\right)+\bar{\theta} \bar{K}\left(a, d, a_{0}\right)\right\} & j=l-1 / 2\end{cases}
\end{aligned}
$$

And

$$
\begin{aligned}
& E_{N 1}=\sqrt{a}(8+2 \mu)+ \\
& +\left\{\begin{array}{c}
2 \pi \theta A_{\mu} S_{e p 1}{ }^{\mu}(\mu=\overline{1,7}) \\
+\pi \frac{\bar{\theta}}{m_{0}} A_{v} S_{e p 1}{ }^{v}(v=\overline{8,10})
\end{array}\right\} j=l+1 / 2 \\
& L_{D}\left\{\begin{array}{c}
2 \pi \theta A_{\mu} S_{e p 1}{ }^{\mu}(\mu=\overline{1,7}) \\
+\pi \frac{\bar{\theta}}{m_{0}} A_{v} S_{e p 1}{ }^{v}(v=\overline{8,10})
\end{array}\right\} j=l-1 / 2
\end{aligned}
$$

From relations (20) and (24), we can write the commutative (CSEP) Hamiltonian $H_{\text {csep }}$ and the generated new spin-orbital interaction $H_{c s e p-s o}$, in (NC-2D: RSP), respectively, as:

$$
\begin{gathered}
H_{\text {csep }}=\left[-\frac{1}{2 m_{0}}\left(\frac{1}{r} \frac{\partial}{\partial r}\left(r \frac{\partial}{\partial r}\right)+\frac{1}{r^{2}} \frac{\partial^{2}}{\partial \phi^{2}}\right)+a r^{2}+\frac{b}{r^{2}}+\frac{c}{r^{4}}+\frac{d}{r^{6}}\right] I_{2 \times 2} \\
H_{c s e p-s o}=\left\{\theta\left(-a+\frac{2 b}{r^{4}}+\frac{2 c}{r^{6}}+\frac{2 d}{r^{8}}\right)+\frac{\bar{\theta}}{2 m_{0}}\right\} \vec{G}^{2}
\end{gathered}
$$

Then, the diagonal matrix of spin-orbit operator which created by the effect of the noncommutative property of the space and phase is given by:

$$
H_{\text {csep-so }}=\left\{2 \theta\left(\frac{b}{r^{4}}+\frac{c}{r^{6}}+\frac{2 d}{r^{8}}-\frac{a}{2}\right)+\frac{\bar{\theta}}{2 m_{0}}\right\}\left(\begin{array}{cc}
L_{U} & 0 \\
0 & L_{D}
\end{array}\right)
$$

Naturally, the operator $H_{\text {csep }}$ represents the interaction of electron with spin $(1 / 2)$ with (SEP) in ordinary $2 \mathrm{D}$ commutative space while the matrix $H_{\text {csep-so }}$ is the spin-orbit interaction induced by the new structure of spaces-phase. The obtained new levels are characterized, in addition to the quantum number $n$, by the new quantum numbers $(\boldsymbol{j}, \boldsymbol{l})$ and $s_{z}= \pm \frac{1}{2}$, contrary to the old commutative levels which are depended only on quantum number $\boldsymbol{n}$.

\subsection{The Modifications to the Energies Levels Produced by Modified Zeeman Effect for Modified (CPIHOIQ) Potential}

Furthermore, it is possible to draw another physical interpretation for the results of the noncommutativity of the spaces-phases for modified (CPIHOIQ) potential in (NC-2D: RSP) if we choose the parameters $(\theta$ and $\bar{\theta})$ and the vector of a magnetic field $(\theta=\sigma B$ and $\bar{\theta}=\bar{\Omega} B$ ) and $\overleftrightarrow{B}=B \vec{k}$, then we can write both $\theta L_{z}$ and $\bar{\theta} L_{z}$ as follows:

$$
\theta L_{z}=\sigma \overleftrightarrow{\jmath} \overleftrightarrow{B}-\sigma H_{Z} \text { and } \bar{\theta} L_{z}=\bar{\Omega} \overleftrightarrow{\jmath} \overleftrightarrow{B}-\bar{\Omega} H_{Z}
$$

Where $\sigma$ and $\bar{\Omega}$ are two real infinitesimal proportionality-constants, the magnetic moment equal spin operator $(\vec{\mu}=\vec{s})$ and $H_{Z}$ is the usual Zeeman field in commutative spaces. Substituting eq. (51) and (20) into eq. (18) leads to the second new NC-2D Hamiltonian $H_{n c-c s e p 2}$ :

$$
\begin{aligned}
& H_{n c-c s e p 2}=-\frac{1}{2 m_{0}}\left(\frac{1}{r} \frac{\partial}{\partial r}\left(r \frac{\partial}{\partial r}\right)+\frac{1}{r^{2}} \frac{\partial^{2}}{\partial \phi^{2}}\right)+ \\
& +a r^{2}+\frac{b}{r^{2}}+\frac{c}{r^{4}}+\frac{d}{r^{6}}+H_{\text {sep-mag }}
\end{aligned}
$$

Where the operator $H_{\text {csep-mag }}$ is given by for modified (CPIHOIQ) potential:

$$
\begin{aligned}
& H_{\text {csep-mag }}=\left(2 \sigma\left(\frac{b}{r^{4}}+\frac{c}{r^{6}}+\frac{d}{r^{8}}-\frac{a}{2}\right)+\frac{\bar{\Omega}}{2 m_{0}}\right) \times \\
& \times\left(-H_{Z}+\overleftrightarrow{J} \overleftrightarrow{B}\right)
\end{aligned}
$$

The above operator represents two interactions between an electron and external magnetic field; the first one is the ordinary Zeeman Effect and the latter is the new interaction coupling between the total monument $\vec{J}$ and external magnetic field $\overleftrightarrow{B}$, a similarly calculations lead to obtain the exact corrections to the energy 
levels $E_{n c-m a g 0}$ and $E_{n c-m a g 1}$ produced by the effect of the modified Zeeman effect which represent by eq. (53) as follows:

$$
\begin{aligned}
& E_{n c-\operatorname{mag} 0}=B m\left\{\sigma K\left(a, d, a_{0}\right)+\bar{\Omega} \bar{K}\left(a, d, a_{0}\right)\right\} \\
& E_{n c-m a g 1}=B m\left\{2 \pi \sigma A_{\mu} S_{e p 1}{ }^{\mu}(\mu=\overline{1,7})+\right. \\
& \left.+\frac{\pi}{m_{0}} \bar{\Omega} A_{v} S_{e p 1}{ }^{v}(v=\overline{8,10})\right\}
\end{aligned}
$$

With $-l \leq m \leq+l$.

\section{RESULTS AND DISCUSSIONS:}

We solved exactly the deformed Schrödinger equation for a fermionic particle under (CSEP) with novel two parts $\left(2 \theta\left(\frac{b}{r^{4}}+\frac{c}{r^{6}}+\frac{d}{r^{8}}-\frac{a}{2}\right)+\frac{\bar{\theta}}{2 m_{0}}\right) L_{z}$. The global specter of energy $E_{n c-s e p 0}$ and $E_{n c-s e p 1}$ for ground state and first excited states can be deduced from Eqs. (11.1), (11.2), (48.1), (48.2) and (54) for modified (CPIHOIQ) potential as follows:

$$
\begin{aligned}
& E_{n c-s e p 0}=\sqrt{a}(4+2 \mu)+B m\left\{\sigma K\left(a, d, a_{0}\right)+\bar{\Omega} \bar{K}\left(a, d, a_{0}\right)\right\} \\
& + \begin{cases}L_{U}\left\{\theta K\left(a, d, a_{0}\right)+\bar{\theta} \bar{K}\left(a, d, a_{0}\right)\right\} & j=l+1 / 2 \\
L_{D}\left\{\theta K\left(a, d, a_{0}\right)+\bar{\theta} \bar{K}\left(a, d, a_{0}\right)\right\} & j=l-1 / 2\end{cases}
\end{aligned}
$$

And

$$
\begin{aligned}
& E_{n c-s e p 1}=\sqrt{a}(8+2 \mu)+B m\left\{2 \pi \sigma A_{\mu} S_{e p 1}{ }^{\mu}(\mu=\overline{1,7})+\right. \\
& \left.+\pi \frac{\bar{\Omega}}{m_{0}} A_{v} S_{e p 1}{ }^{v}(v=\overline{8,10})\right\}+ \\
& +\left\{\begin{array}{l}
L_{U}\left\{2 \pi \theta A_{\mu} S_{e p 1}{ }^{\mu}(\mu=\overline{1,7})+\pi \frac{\bar{\theta}}{m_{0}} A_{v} S_{e p 1}{ }^{v}(v=\overline{8,10})\right\} j=l+1 / 2 \\
L_{D}\left\{2 \pi \theta A_{\mu} S_{e p 1}{ }^{\mu}(\mu=\overline{1,7})+\pi \frac{\bar{\theta}}{m_{0}} A_{v} S_{e p 1}{ }^{v}(v=\overline{8,10})\right\} \\
j=l-1 / 2
\end{array}\right.
\end{aligned}
$$

It's well-known, that the quantum number $m$ can be takes $(2 l+1)$ values and we have also two values for $j=l \pm \frac{1}{2}$, thus every state in usually two dimensional space of modified potential $V_{\text {sep }}(\hat{r})$ will be in (NC-2D: RSP): $2(2 l+1)$ sub-states. Regarding the previous obtained results, we can conclude the global diagonal noncommutative Hamiltonian matrix $H_{n c-s e p}$ of $\operatorname{order}(2 \times 2)$, with elements $\left(H_{n c-s e p}\right)_{11}$ and $\left(H_{n c-s e p}\right)_{22}$ :

$$
\begin{aligned}
& \left(H_{n c-s e p}\right)_{11}=-\frac{1}{2 m_{0}}\left(\frac{1}{r} \frac{\partial}{\partial r}\left(r \frac{\partial}{\partial r}\right)+\frac{1}{r^{2}} \frac{\partial^{2}}{\partial \phi^{2}}\right)+ \\
& +a r^{2}+\frac{b}{r^{2}}+\frac{c}{r^{4}}+\frac{d}{r^{6}}+ \\
& +L_{U}\left\{\theta\left(\frac{2 b}{r^{4}}+\frac{2 c}{r^{6}}+\frac{2 d}{r^{8}}-a\right)+\frac{\bar{\theta}}{2 m_{0}}\right\}+ \\
& +\left(\sigma\left(\frac{2 b}{r^{4}}+\frac{2 c}{r^{6}}+\frac{2 d}{r^{8}}-a\right)+\frac{\bar{\Omega}}{2 m_{0}}\right)\left(-H_{Z}+\breve{J} \vec{B}\right) \\
& \text { if } j=l+\frac{1}{2} \Rightarrow \operatorname{spin} u p
\end{aligned}
$$

And

$$
\begin{aligned}
& \left(H_{n c-s e p}\right)_{22}=-\frac{1}{2 m_{0}}\left(\frac{1}{r} \frac{\partial}{\partial r}\left(r \frac{\partial}{\partial r}\right)+\frac{1}{r^{2}} \frac{\partial^{2}}{\partial \phi^{2}}\right)+ \\
& +a r^{2}+\frac{b}{r^{2}}+\frac{c}{r^{4}}+\frac{d}{r^{6}}+ \\
& +L_{D}\left\{2 \theta\left(\frac{b}{r^{4}}+\frac{c}{r^{6}}+\frac{d}{r^{8}}-\frac{a}{2}\right)+\frac{\bar{\theta}}{2 m_{0}}\right\}+ \\
& +\left\{2 \sigma\left(\frac{b}{r^{4}}+\frac{c}{r^{6}}+\frac{d}{r^{8}}-\frac{a}{2}\right)+\frac{\bar{\Omega}}{2 m_{0}}\right\}\left(-H_{Z}+\overleftrightarrow{J} \overleftrightarrow{B}\right) \\
& \text { if } j=l+\frac{1}{2} \Rightarrow \text { spin up }
\end{aligned}
$$

As it's mentioned in our work [37], the two parameters $\theta$ and $\bar{\theta}$ was played an opposite rolls which allow us to introduce the following gauge fixing condition of Maireche:

$$
\bar{\theta}=-\rho \theta
$$

Here $\rho$ is a positive constant, our obtained spectrum, will be proportioned only to the infinitesimal parameter $\theta$ and positive constant $\rho$ as follows:

$$
\begin{aligned}
& E_{n c-s e p 0}=\sqrt{a}(4+2 \mu)+B m \sigma\left\{K\left(a, d, a_{0}\right)+\rho \bar{K}\left(a, d, a_{0}\right)\right\} \\
& +\theta \begin{cases}L_{U}\left\{K\left(a, d, a_{0}\right)-\rho \bar{K}\left(a, d, a_{0}\right)\right\} & j=l+1 / 2 \\
L_{D}\left\{K\left(a, d, a_{0}\right)-\rho \bar{\theta} \bar{K}\left(a, d, a_{0}\right)\right\} & j=l-1 / 2\end{cases}
\end{aligned}
$$

And

$$
\begin{aligned}
& E_{n c-s e p 1}=\sqrt{a}(8+2 \mu)+\pi B m \sigma \times \\
& \times\left\{2 A_{\mu} S_{e p 1}{ }^{\mu}(\mu=\overline{1,7})-\frac{\rho}{m_{0}} A_{\nu} S_{e p 1}{ }^{v}(v=\overline{8,10})\right\} \\
& +\pi \theta\left\{\begin{array}{l}
L\left(j=l+\frac{1}{2}, l, s=1 / 2\right)\left\{2 A_{\mu} S_{e p 1}{ }^{\mu}(\mu=\overline{1,7})+\frac{\rho}{m_{0}} A_{\nu} S_{e p 1}{ }^{v}(v=\overline{8,10})\right\} \\
L(j=l-1 / 2 \\
j=l-1 / 2
\end{array}\right.
\end{aligned}
$$

And the corresponding (NC-2D: RES) Hamiltonian operator $H_{n c-s e p}$ reduced to the simplest form: 


$$
\begin{aligned}
& \left(H_{n c-c s e p}\right)_{11}=-\frac{1}{2 m_{0}}\left(\frac{1}{r} \frac{\partial}{\partial r}\left(r \frac{\partial}{\partial r}\right)+\frac{1}{r^{2}} \frac{\partial^{2}}{\partial \phi^{2}}\right)+ \\
& +a r^{2}+\frac{b}{r^{2}}+\frac{c}{r^{4}}+\frac{d}{r^{6}}+ \\
& +L_{U} \theta\left\{\frac{2 b}{r^{4}}+\frac{2 c}{r^{6}}+\frac{2 d}{r^{8}}-a-\frac{\rho}{2 m_{0}}\right\}+ \\
& +\sigma\left(\left(\frac{2 b}{r^{4}}+\frac{2 c}{r^{6}}+\frac{2 d}{r^{8}}-a\right)-\frac{\rho}{2 m_{0}}\right) \quad \text { for- spin -up } \\
& \left(-H_{Z}+\overleftrightarrow{J} \overleftrightarrow{B}\right) \quad \text { if } j=l+\frac{1}{2} \quad
\end{aligned}
$$

And

$$
\begin{aligned}
& \left(H_{n c-c s e p}\right)_{22}=-\frac{1}{2 m_{0}}\left(\frac{1}{r} \frac{\partial}{\partial r}\left(r \frac{\partial}{\partial r}\right)+\frac{1}{r^{2}} \frac{\partial^{2}}{\partial \phi^{2}}\right)+ \\
& +a r^{2}+\frac{b}{r^{2}}+\frac{c}{r^{4}}+\frac{d}{r^{6}}+ \\
& +L_{D} \theta\left\{\frac{2 b}{r^{4}}+\frac{2 c}{r^{6}}+\frac{2 d}{r^{8}}-a-\frac{\rho}{2 m_{0}}\right\}+ \\
& +\sigma\left(\frac{2 b}{r^{4}}+\frac{2 c}{r^{6}}+\frac{2 d}{r^{8}}-a-\frac{\rho}{2 m_{0}}\right) \\
& \left(-H_{Z}+\overleftrightarrow{J} \overleftrightarrow{B}\right) \quad \text { if } j=l+\frac{1}{2} \text { for- spin -down }
\end{aligned}
$$

Then, the obtained noncommutative Hamiltonian $H_{n c-s e p}$ depended only on one parameter $\theta$, which allow us to reducing the new symmetries for noncommutative two dimensional real spaces and phases (NC-2D: RSP) for modified (CPIHOIQ) potential to the noncommutative two dimensional real spaces (NC-2D: RS) under above gauge fixing condition of Maireche. Finally, it is worth to mention that the global modified potential (18) can be rewritten as:

$$
V_{\text {sep }}(\hat{r})=a r^{2}+\frac{b}{r^{2}}+\frac{c^{\prime}}{r^{4}}+\frac{d^{\prime}}{r^{6}}+H_{\text {se-newper }}(r)
$$

Where $c^{\prime} \equiv c+2 b \theta L_{z}, \quad d^{\prime} \equiv d+2 b \theta L_{z}$ and the new supplementary term $H_{\text {se-newper }}(r)$ takes the explicitly physical form:

$$
H_{\text {se-newper }}(r)=\theta \frac{2 d}{r^{8}} L_{z}+\left(\frac{\bar{\theta}}{2 m_{0}}-a \theta\right) L_{z}
$$

Can be considered as a new perturbative terms with two translations

$$
c^{\prime} \rightarrow c+2 b \theta L_{z}, d^{\prime} \rightarrow d+2 b \theta L_{z}
$$

In this case the energy eigenvalue of the (CSEP) corresponding ground state and first excited states are changed and replace by two new values $E_{0 \text { sep }}$ and $E_{1 \text { sep }}$ :

$$
\begin{aligned}
& E_{0 \text { sep }}=\sqrt{a}\left(4+\frac{c+2 b \theta L_{z}}{\sqrt{d}}\right)=\sqrt{a}\left(4+\frac{c}{\sqrt{d}}\right)+2 \sqrt{\frac{a}{d}} b \theta m \\
& E_{1 \text { sep }}=\sqrt{a}\left(8+\frac{c}{\sqrt{d}}\right)+2 \sqrt{\frac{a}{d}} b \theta m
\end{aligned}
$$

And the new normalized eignenfunctions $\Psi_{\text {sep }}{ }^{(0)}(\vec{r})$ and $\Psi_{\text {sep }}{ }^{(1)}(\vec{r})$ are determined by new forms:

$$
\Psi_{\text {sep }}^{(0)}(\ddot{r})=a_{0} r^{\delta^{\prime}-\frac{1}{2}} \exp \left(-\frac{\sqrt{a} r^{2}+\sqrt{d^{\prime}} r^{-2}}{2}\right)
$$

And

$$
\begin{aligned}
& \Psi_{\text {sep }}^{(1)}(\stackrel{\leftrightarrow}{r})=\left(a_{0}+a_{1} r^{2}\right) r^{\delta^{\prime}-\frac{1}{2}} \times \\
& \times \exp \left(-\frac{\sqrt{a} r^{2}+\sqrt{d} \cdot r^{-2}}{2}\right) \exp ( \pm i \phi)
\end{aligned}
$$

Where $\delta^{\prime}=\frac{3}{2}+\mu^{\prime}$ and $\mu^{\prime}=\mu+\frac{b}{\sqrt{d}} \theta m$, know we can determine the expectation value of $\left\langle\frac{1}{r^{8}}\right\rangle_{\Psi_{\text {sep }}{ }^{(0)}(\vec{r})}$ and $\left\langle\frac{1}{r^{8}}\right\rangle_{\Psi_{s e p}^{(1)}(\vec{r})}$ as follows:

$$
\begin{aligned}
& \left\langle\frac{1}{r^{8}}\right\rangle_{\Psi_{s e p}^{(0)}(\bar{r})}=a_{0}^{2} \int_{0}^{+\infty} r^{\left(2 \delta^{\prime}-7\right)-1} \exp \left(-\beta r^{2}-\gamma^{\prime} r^{-2}\right) d r \\
& \left\langle\frac{1}{r^{8}}\right\rangle_{\Psi_{s e p}^{(1)}(\bar{r})}=\int_{0}^{+\infty}\left(\begin{array}{l}
a_{0}{ }^{2} r^{\left(2 \delta^{\prime}-7\right)-1} \\
+a_{1}{ }^{2} r^{\left(2 \delta^{\prime}-3\right)-1} \\
+2 a_{0} a_{1} r^{\left(2 \delta^{\prime}-5\right)-1}
\end{array}\right) \exp \left(-\beta r^{2}-\gamma^{\prime} r^{-2}\right) d r
\end{aligned}
$$

Where $\gamma^{\prime} \equiv \sqrt{d^{\prime}}$, a simple calculation gives:

$$
\begin{aligned}
& \left\langle\frac{1}{r^{8}}\right\rangle_{\Psi_{s e p}(0)(\vec{r})}=2 \pi a_{0}^{2}\left(\frac{\gamma^{\prime}}{\beta}\right)^{\frac{2 \delta^{\prime}-7}{4}} K_{\left(2 \delta^{\prime}-7\right) / 2}\left(2 \sqrt{\beta \gamma^{\prime}}\right) \\
& \left\langle\frac{1}{r^{8}}\right\rangle_{\Psi_{s e p}^{(1)}(\vec{r})}=2 \pi\left|a_{0}\right|^{2}\left(\frac{\gamma^{\prime}}{\beta}\right)^{\frac{2 \delta^{\prime}-7}{4}} K_{\left(2 \delta^{\prime}-7\right) / 2}\left(2 \sqrt{\beta \gamma^{\prime}}\right)+ \\
& +2 \pi\left|a_{1}\right|^{2}\left(\frac{\gamma^{\prime}}{\beta}\right)^{\frac{2 \delta^{\prime}-3}{4}} K_{\left(2 \delta^{\prime}-3\right) / 2}\left(2 \sqrt{\beta \gamma^{\prime}}\right)+ \\
& +4 \pi a_{0} a_{1}\left(\frac{\gamma^{\prime}}{\beta}\right)^{\frac{2 \delta^{\prime}-5}{4}} K_{\left(2 \delta^{\prime}-5\right) / 2}\left(2 \sqrt{\beta \gamma^{\prime}}\right)
\end{aligned}
$$

The energy contributions $\left\langle H_{2 p e r}\right\rangle_{0}$ and $\left\langle H_{2 p e r}\right\rangle_{1}$ produced by the second term of perturbation operator $H_{\text {se-newper }}(r)$ are given by, respectively:

$$
\begin{gathered}
\left\langle H_{2 p e r}\right\rangle_{0}=\left(\frac{\bar{\theta}}{2 m_{0}}-a \theta\right) m\left|a_{0}\right|^{2} \times \\
\times \int_{0}^{+\infty} r^{\left(2 \delta^{\prime}+1\right)-1} \exp \left(-\beta r^{2}-\gamma^{\prime} r^{-2}\right) d r \\
\left\langle H_{2 p e r}\right\rangle_{1}=\left(\frac{\bar{\theta}}{2 m_{0}}-a \theta\right) m \int_{0}^{+\infty}\left(\begin{array}{l}
a_{0}^{2} r^{\left(2 \delta^{\prime}+1\right)-1} \\
+a_{1}^{2} r^{\left(2 \delta^{\prime}+5\right)-1} \\
+2 a_{0} a_{1} r^{\left(2 \delta^{\prime}+3\right)-1}
\end{array}\right) \times \\
\times \exp \left(-\beta r^{2}-\gamma^{\prime} r^{-2}\right) d r
\end{gathered}
$$


We apply the special integral (35) to obtain $\left\langle H_{2 p e r}\right\rangle_{0}$ and $\left\langle H_{2 \text { per }}\right\rangle_{1}$, respectively, as follows:

$$
\begin{aligned}
& \left\langle H_{2 p e r}\right\rangle_{0}=2 \pi\left(\frac{\bar{\theta}}{2 m_{0}}-a \theta\right) m\left|a_{0}\right|^{2}\left(\frac{\gamma^{\prime}}{\beta}\right)^{\frac{2 \delta^{\prime}+1}{4}} \times \\
& \times K_{\left(2 \delta^{\prime}+1\right) / 2}\left(2 \sqrt{\beta \gamma^{\prime}}\right)
\end{aligned}
$$

And

$$
\begin{aligned}
& \left\langle H_{2 p e r}\right\rangle_{1}=2 \pi\left(\frac{\bar{\theta}}{2 m_{0}}-a \theta\right) \times \\
& \times\left\{\begin{array}{l}
a_{0}^{2}\left(\frac{\gamma^{\prime}}{\beta}\right)^{\frac{2 \delta^{\prime}+}{4}} K_{\left(2 \delta^{\prime}+1\right) / 2}\left(2 \sqrt{\beta \gamma^{\prime}}\right) \\
+a_{1}^{2}\left(\frac{\gamma^{\prime}}{\beta}\right)^{\frac{2 \delta^{\prime}+5}{4}} K_{\left(2 \delta^{\prime}+5\right) / 2}\left(2 \sqrt{\beta \gamma^{\prime}}\right) \\
+2 a_{0} a_{1}\left(\frac{\gamma^{\prime}}{\beta}\right)^{\frac{2 \delta^{\prime}+3}{4}} K_{\left(2 \delta^{\prime}+3\right) / 2}\left(2 \sqrt{\beta \gamma^{\prime}}\right)
\end{array}\right\} m
\end{aligned}
$$

The obtained results (66), (69) and (70) allow us to getting the total energy produced by the modified (SEP) potential in Noncommutative Spaces and Phases Symmetries. It is important to notice, the appearance of the

\section{REFERENCES}

1. M.S. Child, M.S. Shi-Hai Dong, X.G. Wang, J. Phys. A 33 No 32, 5653 (2000).

2. S.K. Bose, Il Nuovo Cimento B 109 No 11, 1217 (1994).

3. L. Buragohain L, S.A.S. Ahmed, Lat. Am. J Phys. Educ. 4 No 1,79 (2010).

4. S.A.S. Ahmed, B.C. Borah, D. Sarma, Eur. Phys. J. D 17 No 1,5 (2001).

5. S.M. Ikhdair, R. Sever, CEJP 5 No 4, 516 (2007).

6. M.M. Nieto, Am. J. Phys. 47, 1067 (1979).

7. S.M. Ikhdair, R. Sever, J. Mol. Structur. Theochem. 806, 155 (2007).

8. S.A.S. Ahmed, L. Buragohain, Phys. Scr. 80 No 2, 4 (2009).

9. S.K. Bose, N. Gupta, Nuovo Cimento B 113, 299 (1998).

10. G.P. Flesses, A. Watt, J. Phys. A: Math. Gen. 14 No 9, L315 (1981).

11. S.M. Ikhdair, R. Sever, Ann. Phys. (Leipzig) 16 No 3, 218 (2007).

12. Shi-Hai Dong, Phys. Scr. 64 No 4, 273 (2001).

13. Shi-Hai Dong, Z.Q. Ma, J. Phys. A 31 No 49, 9855 (1998).

14. Shi-Hai Dong, Int. J. Theor. Phys. 40 No 2, 559 (2001).

15. A.A. Rajabi, M. Hamzavi, J. Theoret. Appl. Phys. 7, 17 (2013).

16. S.M. Ikhdair, R. Sever, Adv. High Energy Phys. 2013 , ID 562959 (2013).

17. Shi-Hai Dong, G.H. San, Found. Phys. Lett. 16 No 4, 357 (2003).

18. L. Buragohain, S.A.S. Ahmed, Lat. Am. J. Phys. Educ. 4 No 1,79 (2010).

19. S.M. Ikhdair, J. Mod. Phys. 3 No 2, 170 (2012).

20. H. Hassanabadi, E. Maghsoodi, R. Oudi, S. Zarrinkamar, H. Rahimov, Eur. Phys. J. Plus. 127, 120 (2012).

21. H. Hassanabadi, M. Hamzavi, S. Zarrinkamar, A.A. Rajabi, Int. J. Phys. Sci. 6 No 3, 583 (2011).

22. Shi-Hai Dong, Z.Q. Ma, G. Esposito, Found. Phys. Lett. 12 No 5, 465 (1999). polarization states of a fermionic particle for modified (CPIHOIQ) potential indicates the validity of the results in the field of high energy where the relativistic Dirac equations is applied, which allowing to apply these results of various Nano-particles at Nanoscales

\section{CONCLUSIONS REMARKS}

This recent work has found a theoretical solution for the energy spectrum of modified singular even-power potential in noncommutative two dimensional spacesphases based to Dong Shi-Hai work in reference [25] by using Boopp's shift method and standard perturbation theory instead to solving directly Schrödinger equation with star product. We show that the obtained new results are degenerated and different totally to original results in ordinary commutative space. Due the useful obtained results to this modified potential on the atomics and optical physics, we hope to discover other physical applications for the obtained corrections to the energy and the corresponding noncommutative Hamiltonian for (MSEP) potential is also constructed.

\section{ACKNOWLEDGMENTS}

This work was supported with search laboratory of Physics and Material Chemistry in University of M'sila, Algeria.

23. Gupta Pramila, Mehrotra Indira, J. Modern Phys. 3, 1530 (2012).

24. Shi-Hai Dong, Guo-Hua Sun, Phys. Scr. 70, 94 (2004)

25. D. Shi-Hai, Int. J. Theor. Phys. 39 No 4, 1119 (2000).

26. A.O. Brut, J. Math. Phys. 21, 568 (1980).

27. B.H. Bransden, C.J. Joachain, Physics of Atoms and Molecules (Longman: London: 1983).

28. D.T. Jacobus, Department of Physics, Stellenbosch University, South Africa (2010).

29. Z.H. Yang, C.Y. Long, S.J. Qin, Z.W. Long, Int. J. Theor. Phys. 49 No 3, 644 (2010).

30. Y. Yuan, L. Kang, W. Jian-Hua, C. Chi-Yi, Chin. Phys. C 34 No 5, 543 (2010).

31. B. Mirza, R. Narimani, S. Zare, Commun. Theor. Phys. 55 , 405 (2011).

32. A. Maireche, Life Sci. J. 11 No 6, 353 (2014).

33. A. Maireche, Afr. Rev. Phys. 9, 479 (2014).

34. A. Maireche, J. Nano-Electron. Phys. 7 No 2, 02003 (2015)

35. A. Maireche, Afr. Rev. Phys. 9, 185 (2014).

36. A. Maireche, Afr. Rev. Phys. 10:0014, 97 (2015).

37. A. Maireche, Int. Lett. Chem., Phys. Astronomy 56, 1 (2015).

38. A. Maireche, Int. Lett. Chem., Phys. Astronomy 60, 11 (2015).

39. A. Maireche, Afr. Rev. Phys. 10:0025, 177 (2015)

40. A. Maireche, Afr. Rev. Phys. 10:0046, 373 (2015).

41. A. Maireche, Int. Lett. Chem., Phys. Astronomy, 58, 164 (2015).

42. A. Maireche, Med. J. Model. Simul. 04, 060 (2015)

43. A. Maireche, Int. Lett. Chem., Phys. Astronomy, 61, 38 (2015).

44. A. Maireche, J. Nano- Electron. Phys. 7 No 4, 04021 (2015).

45. A. Maireche, J. Nano- Electron. Phys. 7 No 3, 03047 (2015). 
46. A. Maireche, Lat. Am. J. Phys. Educ. 9 No 1, 1301 (2015). 47. A. Maireche, Nano World J. 1 No 4, 120 (2016).

48. A. Maireche, J. Nano- Electron. Phys. 8 No 1, 01020 (2016). 49. A. Maireche, J. Nano- Electron. Phys. 8 No 1, 01021 (2016).

50. S. Cai, T. Jing, G. Guo, R. Zhang, Int. J. Theor. Phys. 49 No 8, 1699 (2010).

51. J. Lee, J. Korean Phys. Soc. 47 No 4, 571 (2005).

52. A. Jahan, Braz. J. Phys. 38, 144 (2008).

53. A.E.F. Djemei, H. Smail, Commun. Theor. Phys. 41, 837 (2004).
54. A. Smailagic, E. Spallucci, Phys. Rev. D 65, 107701 (2002). 55. H. Snyder, Phys. Rev. 71, 38 (1947).

56. Altug Arda, Ramazan Server, Commun. Theor. Phys. 58 No 1, 27 (2012).

57. M. Abramowitz, I.A. Stegun, Handbook of Mathematical Functions with Formulas, Graphs, and Mathematical Tables (Dover Publications: 1965).

58. I.S. Gradshteyn, I.M. Ryzhik, Table of Integrals, Series and Products, $7^{\text {th }}$ Ed. (Elsevier: 2007). 